\title{
Measurement of Circumferential Liquid Film Based on LIF and Virtual Stereo Vision Sensor
}

\author{
Ting Xue, Xiaokang Lin, and Liuxiangzi Yang \\ College of Electrical Engineering and Automation, Tianjin University, Tianjin 300072, China \\ Correspondence should be addressed to Ting Xue; xueting@tju.edu.cn
}

Received 27 May 2016; Revised 25 July 2016; Accepted 26 July 2016

Academic Editor: Lei Yuan

Copyright (C) 2016 Ting Xue et al. This is an open access article distributed under the Creative Commons Attribution License, which permits unrestricted use, distribution, and reproduction in any medium, provided the original work is properly cited.

\begin{abstract}
Gas-liquid annular flow is widely used in many industrial applications such as petroleum, chemical, and nuclear engineering. The feature parameters of liquid film in the annular flow are of great significance to understand the flow characteristics and measure the flow precisely. For the annular flow, the circumferential features of liquid film are more important than the axial features to acquire abundant flow structures and reveal the flow mechanism. In the paper, a measurement platform based on the laser-induced fluorescence (LIF) and virtual stereo vision sensor is presented. The virtual stereo vision sensor comprises a high-speed camera and two optical reflection sets, which can acquire the liquid film from two views simultaneously and reconstruct the features of liquid film. Image processing techniques are proceeded with to extract the feature parameters of liquid film; then the circumferential flow characteristic can be reconstructed by views transformation and fusion. The flow characteristic based on the thickness distribution is analysed. The experimental results show that the method is valid and effective, which can give a more detailed and accurate description for the liquid film in annular flows.
\end{abstract}

\section{Introduction}

The annular flow is one of the important flow patterns in gasliquid two-phase flow. The flow pattern is characterized by the presence of a thin liquid film flowing along the wall of the tube and surrounding a fast-moving gas core. The core may bring the entrainment of liquid droplets at the high gas velocity, and the flow rate of the gas and liquid phase can vary a lot in the annular flow. Due to the low power consumption, high coefficient of mass transfer, and heat flux density, gas-liquid annular flow is widely used in many industrial applications such as petroleum, chemical, and nuclear engineering and wet natural gas transportation. Due to high quality of the exchange efficiency, the flow pattern is widely used in various industrial productions. The feature parameters of liquid film in the annular flow are of great significance to understand the flow mechanism precisely [1].

The characteristic parameters of annular flow include the thickness of the liquid film, wave velocity, wave height, and shear force, and the liquid film thickness is the basic and most important parameter to be measured accurately [2]. There are many methods that have been developed to investigate the liquid film in gas-liquid annular flow. Among the various detection sensors, the measurement methods are generally divided into the contact techniques and the noncontact techniques. Han et al. [3] used a parallel wire probe to collect the film thickness time-trace measurements in a small vertical pipe. The method based on the conductance measurement of the liquid film thickness is simple and reliable, but it only can achieve a single local point measurement and may interfere with the flow field. Then many sensors are developed to achieve the multiple points measurement [4-7]. Based on the circumferential array of conductance probes, Andreussi et al. [8] measured the thickness distribution around the pipe wall of a liquid layer flowing in near horizontal pipes. Belt et al. [9] developed a nonintrusive conductivity-based technique to measure the film thickness at 32 positions around the circumference and 10 positions in the axial direction. Mouza et al. [10] measured the time-averaged and instantaneous local film thickness in flowing liquid based on the absorption of light passing through a layer of dyed liquid and the sensitive light detection sensor. Recently, Han et al. [11] measured the averaged annular liquid film thickness by laser confocal displacement meter (LCDM). Based on the digital image 


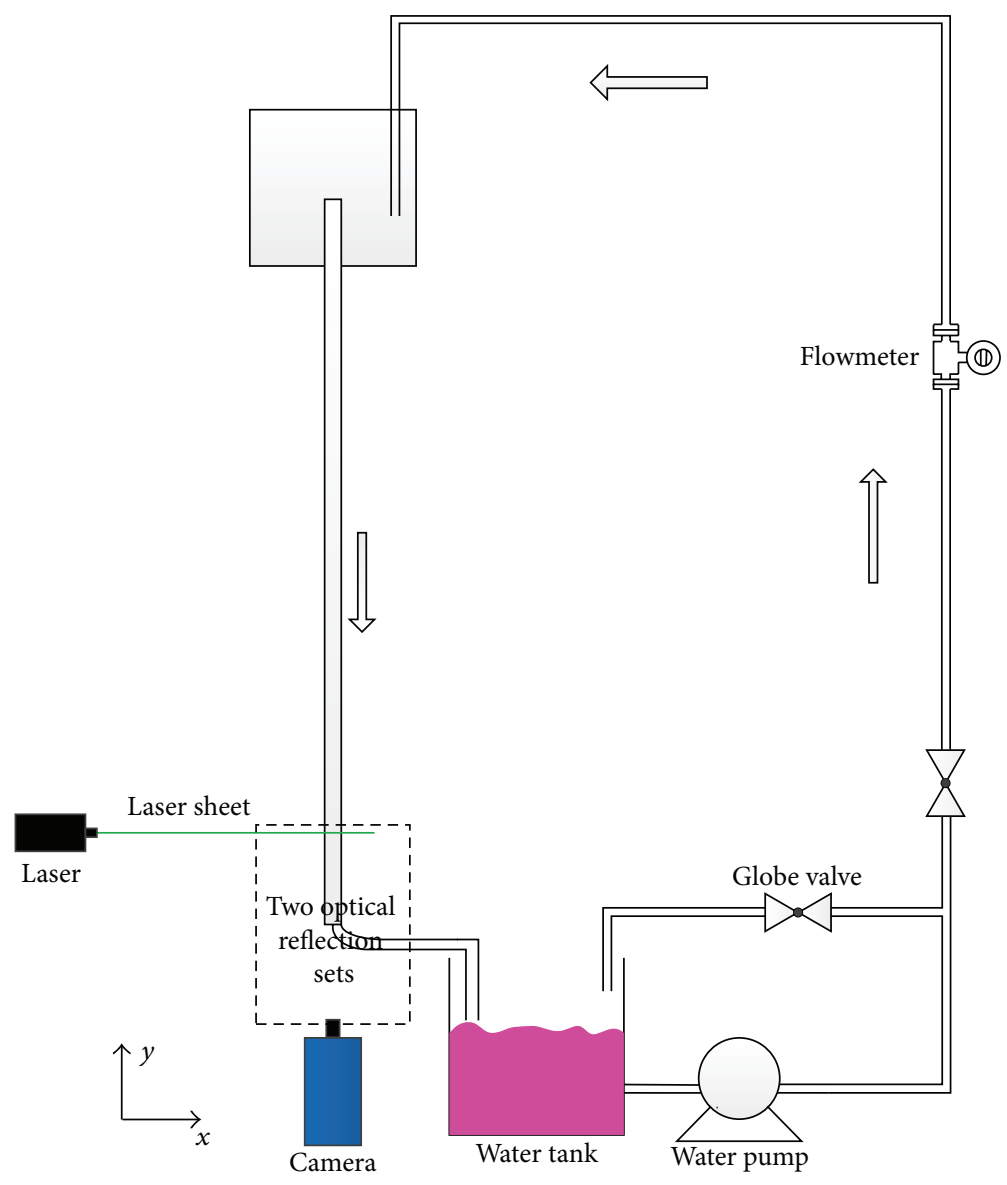

FIgURE 1: Experimental flow loop.

processing and planar laser-induced fluorescence (PLIF) technique, the target images taken by the high-speed camera can be quite a contrast to the background. Alekseenko et al. [12] studied the wavy structure of liquid film in downward annular gas-liquid flow with LIF technique, and the liquid film thickness measurements are resolved in the longitudinal based on the brightness model. Based on the PLIF technique, Kokomoor and Schubring [13] developed a nonintrusive optical technique to provide the film thickness distribution by data reduction algorithms. Zadrazil and Markides [14] studied experimentally the hydrodynamics of downwards gas-liquid annular flows and falling films in a pipe based on the PLIF and Particle Image Velocimetry (PIV). For the annular flow, the cross-sectional images of the liquid film are more important than the longitudinal images to reveal the unsymmetrical flow characteristic of interfacial wave and flow mechanism, especially under high pressure, but the circumferential measurement is also more difficult to achieve. Generally, the two cameras can be employed to observe the liquid film of the cross section in the two different views and reconstruct the circumferential images.

The paper aims to reconstruct the circumferential liquid film of vertical air-water annular flow and extract the characteristic parameter of the liquid film thickness based on the laser-induced fluorescence (LIF) and virtual stereo vision sensor. The virtual stereo vision sensor comprises a high-speed camera and two optical reflection sets, which can acquire the liquid film from two views simultaneously and reconstruct the multiple features of liquid film. A series of image processing techniques are also proceeded with, and the circumferential flow characteristics are reconstructed by views transformation and fusion to analyse the flow characteristic based on the thickness distribution.

The paper is organized as follows: Section 2 introduces the experimental flow loop and develops a virtual stereo vision sensor to achieve the cross-sectional views of liquid film. Section 3 describes the image processing and the feature parameter measurement of the liquid film. In Section 4, we present validation of the liquid film measurement result and the analysis, which is followed by a final conclusion in Section 5.

\section{Experimental Facility}

2.1. Flow Loop. Figure 1 shows a schematic view of the test section to measure the annular liquid film on the cross section of the test pipe. The test and connection pipe was fabricated from Plexiglass and installed vertically. Water was pumped into the upper tank through a pump and then fell downwards into a $25 \mathrm{~mm}$ diameter and $5 \mathrm{~mm}$ thickness test pipe to 
form annular flow. The flow rate of water was controlled by the globe valves and measured by the electromagnetic flow meter with $\pm 0.5 \%$ measurement uncertainty. The virtual stereo vision sensor was fixed at the lower end of the pipe and perpendicular to the $x-y$ plane to measure the fully developed falling films. In the experiment, a high-speed camera of Weinberger MiniVis E2 series coupled with a $35 \mathrm{~mm}$ lens of Nikkor was used. To achieve the instantaneous liquid film on the whole cross section, the high-speed camera is set at a resolution of $960 \times 720$ pixels at the frame rate of $500 \mathrm{~Hz}$.

Due to the monochrome, directivity, high intensity, and high measurement resolution and accuracy, the laser is widely used for many optical diagnoses. In the paper, the planar laser-induced fluorescence technique (LIF) is employed to provide time-resolved images of the liquid film in vertical annular air-water flows. Rhodamine B was dissolved in the flow field as the fluorescent agent based on their specific molecular structure. The laser (NanoLine, Canada) with the wavelength of $532 \mathrm{~nm}$ projects a laser sheet to irradiate the cross section of the test pipe and stimulate the Rhodamine B fluorescence. Then the high speed camera captured the fluorescence signal of liquid film and processed by a series image processing.

2.2. Virtual Stereo Vision Sensor. The measurement scheme of liquid film based on the virtual stereo vision sensor is shown in Figure 2. The optical centre of camera overlaps the central axis of the pipe, and the laser sheet is perpendicular to the optical axis of the camera. A virtual stereo vision sensor is composed of a high-speed camera and two optical reflection sets, and the two optical reflection sets are between the camera and the pipe. Xue et al. [15] developed a virtual binocular stereo vision system to reconstruct the threedimensional morphological parameters and track the bubbles in the bubbly flow. In the paper, it is optimized to view nearly half of cross-sectional liquid film rather than the same point on the two views according to the mathematical model and lots of stimulations.

The virtual binocular stereo vision sensor is employed to capture the two-dimensional images of the liquid film in the left and right image plane of the camera at the same time. The camera is reflected by the reflection mirror group, which can be mirrored to two virtual cameras $C_{2 L}$ and $C_{2 R}$. The two virtual cameras are symmetrical with each other, and there is an optimized angle between the virtual and the real cameras based on the effective FOV (Field of View) and spatial resolution. Therefore, the high-speed camera can view the circumferential liquid film from the two directions. When the images are captured by a single high-speed camera containing the two parts of cross-sectional liquid film, they can be processed by a series of digital image processing and reconstructed as a complete cross-sectional liquid film image.

In the virtual stereo vision sensor, there are two important angle parameters, which are the angle $\alpha$ of the centre mirror and the vertical distance from the optical centre and the angle $\gamma$ between the centre mirror and the same side refraction mirror group. The angle $\alpha$ is optimized by $45^{\circ}$ and $\gamma$ is $20^{\circ}$ eventually. Taking the left optical path as an example, the

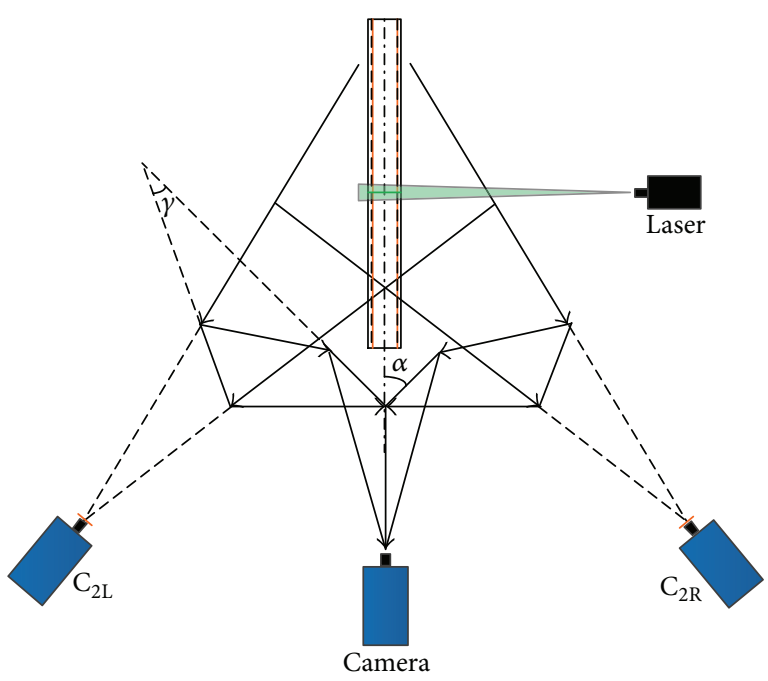

FIGURE 2: Schematic illustration of the virtual stereo vision.

measured object is reflected by the centre mirror and the left reflection mirror, and the angle of the virtual image of the measured object in the vertical direction is $40^{\circ}$, the same as the right side of the optical reflection set.

\section{Image Processing}

A series of image processing and reconstruction procedures should be developed to extract the feature parameters of liquid film based on the virtual stereo vision sensor.

\subsection{Circumferential Images Processing Based on Virtual Stereo} Vision Sensor. A typical instantaneous original image of liquid film captured by the virtual stereo vision sensor is shown in Figure 3(a). Simultaneously, the background image is captured in the absence of water cycle, and the image gray is proceeded with for the original and background image, respectively, and followed by the image subtraction, which can remove the noise due to the ambient light and the defects of the flow pipe effectively. Figure 3(b) shows the anticoloured image after median filter, which uses a $3 * 3$ template to remove the single-pixel noise and maintain the edge of liquid film. We can see that there are two ring sectors in the left part of Figure 3(b). The bigger sector represents the left cross-sectional film, while the smaller one is the right crosssectional film, which overlaps the refraction distortion of left cross-sectional film and the pipe wall and is discarded for the characteristics extraction.

According to the analysis of the virtual stereo vision sensor in Section 2.2, the circumferential liquid film of the pipe was viewed from a certain angle, and it is necessary to proceed with the $40^{\circ}$ projection transformation to simplify the subsequent fusion and reconstruction. The binarized image after projection transformation can be seen in Figure 3(c). Due to the effect of pipe refraction, the liquid film is not the standard semicircle and a calibration should be performed to form an instantaneous cross section image of liquid film. In order to reconstruct the cross-sectional liquid 


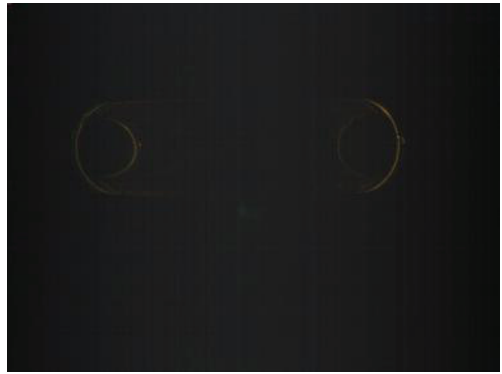

(a) Original image

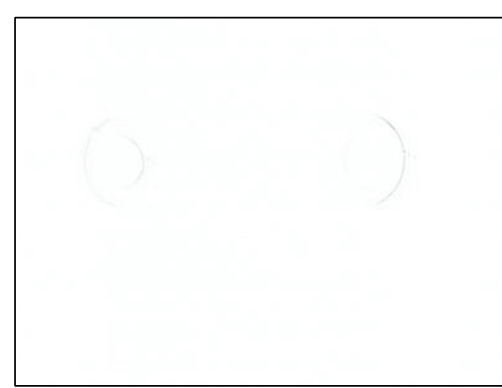

(b) Image after median filter

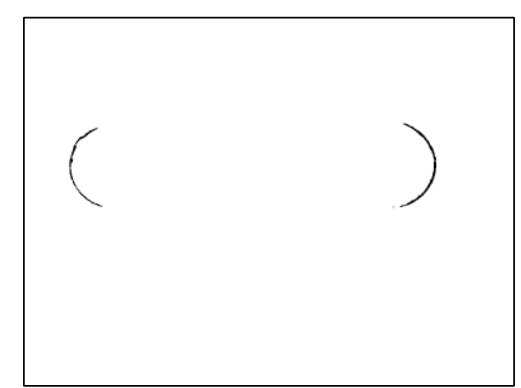

(c) Image after projection transformation

Figure 3: Circumferential image processing, (b) and (c) are anticoloured.

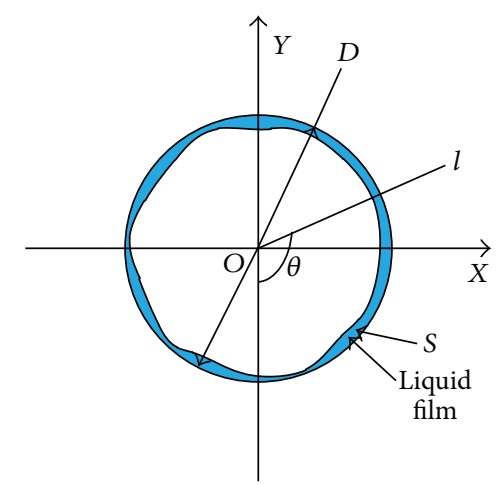

FIgURE 4: Liquid film thickness extraction.

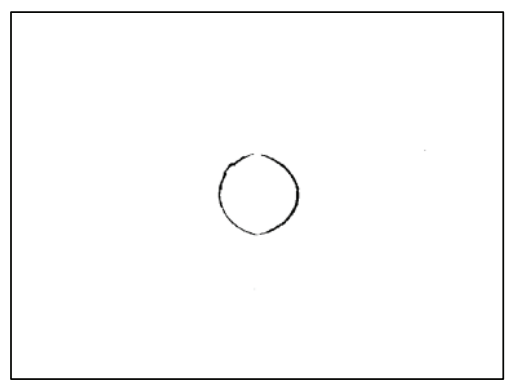

(a) $t=t_{0}$

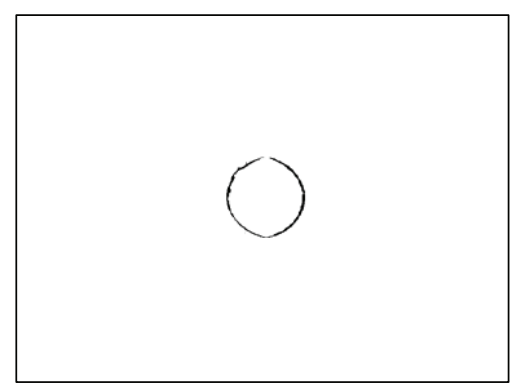

(b) $t=t_{1}$

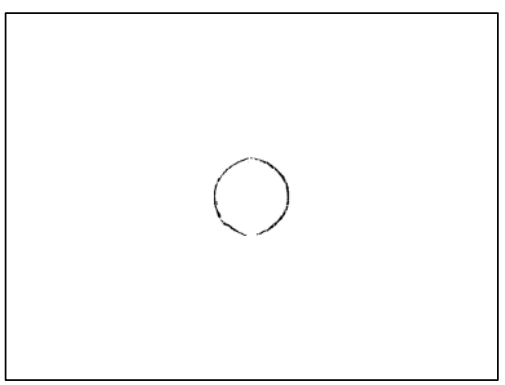

(c) $t=t_{2}$

FIGURE 5: Reconstructed image sequence, (a)-(c) are anticoloured.

film, the centre position of the calibration target should be recorded, which is a guide to reconstruct the two sectors of the liquid film and obtain a whole annular cross section, as shown in Figure 5.

3.2. Characteristic Parameter Extraction. The characteristic parameters of the liquid film can be extracted based on the above reconstructed images. Figure 4 shows the extraction scheme of the liquid film thickness. The centre of the annular liquid film is denoted as the origin of the coordinate system $O$, the angle between the line $l$ and the negative $Y$-axis is defined as $\theta$, and $D$ is the inner diameter of the pipe, that is, the average outer circle diameter in the reconstructed images. With the change of $\theta$, the equation of the line $l$ is transformed.
The liquid film thickness at the $l$ direction can be extracted based on a resolution of $0.154 \mathrm{~mm} /$ pixel. Therefore, the circumferential distribution of the film thickness can be extracted. The circumferential average thickness of liquid film can be given as follows:

$$
\delta=k\left(\frac{D}{2}-\sqrt{\left(\frac{D}{2}\right)^{2}-\frac{S}{\pi}}\right)
$$

where $\delta$ is measured thickness in millimetres, $k$ is calibration parameter, relating the pixel coordinate in image plane and the real distance, and $S$ is the area of the circumferential liquid film in the reconstructed image with the unit of pixel. 


\section{Results and Analysis}

All the experiments were performed with the $25 \mathrm{~mm}$ inner diameter downward pipe, which is described in Section 2. Cross-sectional views of the liquid film are captured at $500 \mathrm{~Hz}$, and the image exposure time is about $2 \mathrm{~ms}$. According to a series of image processing and feature parameters extraction, the high-quality instantaneous images of the liquid film can be reconstructed and are available for analysis.

Figure 5 shows three samples of the reconstructed liquid film images at different time. The average film thickness of the image sequence is $0.55 \mathrm{~mm}, 0.53 \mathrm{~mm}$, and $0.427 \mathrm{~mm}$, respectively. The selected image sequence shows that the annular flow is stable, and the liquid film has a little fluctuation and similar circumferential distribution.

\section{Conclusions}

In the paper, an optical technique based on the laserinduced fluorescence (LIF) and virtual stereo vision sensor is presented to measure the circumferential liquid film in annular flow. The laser-induced fluorescence was applied to separate the intensity of the liquid film in and out of the experimental cross section. A virtual stereo vision sensor is developed to capture the fluorescence image of the whole cross-sectional liquid film. Particularly a series of image processing is developed to extract the feature parameters of liquid film and reconstruct the annular liquid film images. The quantitative thickness features of liquid film are analysed, and the experimental results show that the method is valid and effective, which can give a more detailed characterization of the liquid film to reveal the flow structures and flow mechanism.

\section{Competing Interests}

The authors declare that they have no competing interests.

\section{Acknowledgments}

The authors gratefully acknowledge the support by the National Natural Science Foundation of China (60902084, 61372143).

\section{References}

[1] M. J. Da Silva, T. Sühnel, E. Schleicher, R. Vaibar, D. Lucas, and U. Hampel, "Planar array sensor for high-speed component distribution imaging in fluid flow applications," Sensors, vol. 7, no. 10, pp. 2430-2445, 2007.

[2] M. Damsohn and H.-M. Prasser, "High-speed liquid film sensor for two-phase flows with high spatial resolution based on electrical conductance," Flow Measurement and Instrumentation, vol. 20, no. 1, pp. 1-14, 2009.

[3] H. W. Han, Z. F. Zhu, and K. Gabriel, "A study on the effect of gas flow rate on the wave characteristics in two-phase gas-liquid annular flow," Nuclear Engineering and Design, vol. 236, no. 24, pp. 2580-2588, 2006.
[4] S. Thiele, M. J. Da Silva, and U. Hampel, "Capacitance planar array sensor for fast multiphase flow imaging," IEEE Sensors Journal, vol. 9, no. 5, pp. 533-540, 2009.

[5] M. Demori, V. Ferrari, D. Strazza, and P. Poesio, "A capacitive sensor system for the analysis of two-phase flows of oil and conductive water," Sensors and Actuators A: Physical, vol. 163, no. 1, pp. 172-179, 2010.

[6] X. Hu and W. Yang, "Planar capacitive sensors-designs and applications," Sensor Review, vol. 30, no. 1, pp. 24-39, 2010.

[7] D. Schubring, T. A. Shedd, and E. T. Hurlburt, "Studying disturbance waves in vertical annular flow with high-speed video," International Journal of Multiphase Flow, vol. 36, no. 5, pp. 385-396, 2010.

[8] P. Andreussi, E. Pitton, P. Ciandri et al., "Measurement of liquid film distribution in near-horizontal pipes with an array of wire probes," Flow Measurement and Instrumentation, vol. 47, pp. 7182, 2016.

[9] R. J. Belt, J. M. C. Van't Westende, H. M. Prasser, and L. M. Portela, "Time and spatially resolved measurements of interfacial waves in vertical annular flow," International Journal of Multiphase Flow, vol. 36, no. 7, pp. 570-587, 2010.

[10] A. A. Mouza, N. A. Vlachos, S. V. Paras, and A. J. Karabelas, "Measurement of liquid film thickness using a laser light absorption method," Experiments in Fluids, vol. 28, no. 4, pp. 355-359, 2000.

[11] Y. Han, H. Kanno, Y.-J. Ahn, and N. Shikazono, "Measurement of liquid film thickness in micro tube annular flow," International Journal of Multiphase Flow, vol. 73, pp. 264-274, 2015.

[12] S. V. Alekseenko, A. V. Cherdantsev, O. M. Heinz, S. M. Kharlamov, and D. M. Markovich, "Analysis of spatial and temporal evolution of disturbance waves and ripples in annular gas-liquid flow," International Journal of Multiphase Flow, vol. 67, pp. 122-134, 2014.

[13] W. Kokomoor and D. Schubring, "Improved visualization algorithms for vertical annular flow," Journal of Visualization, vol. 17, no. 1, pp. 77-86, 2014.

[14] I. Zadrazil and C. N. Markides, "An experimental characterization of liquid films in downwards co-current gas-liquid annular flow by particle image and tracking velocimetry," International Journal of Multiphase Flow, vol. 67, pp. 42-53, 2014.

[15] T. Xue, L. Qu, Z. Cao, and T. Zhang, “Three-dimensional feature parameters measurement of bubbles in gas-liquid two-phase flow based on virtual stereo vision," Flow Measurement and Instrumentation, vol. 27, no. 10, pp. 29-36, 2012. 


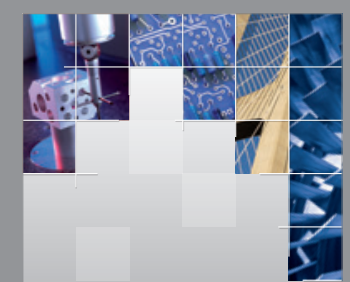

\section{Enfincering}
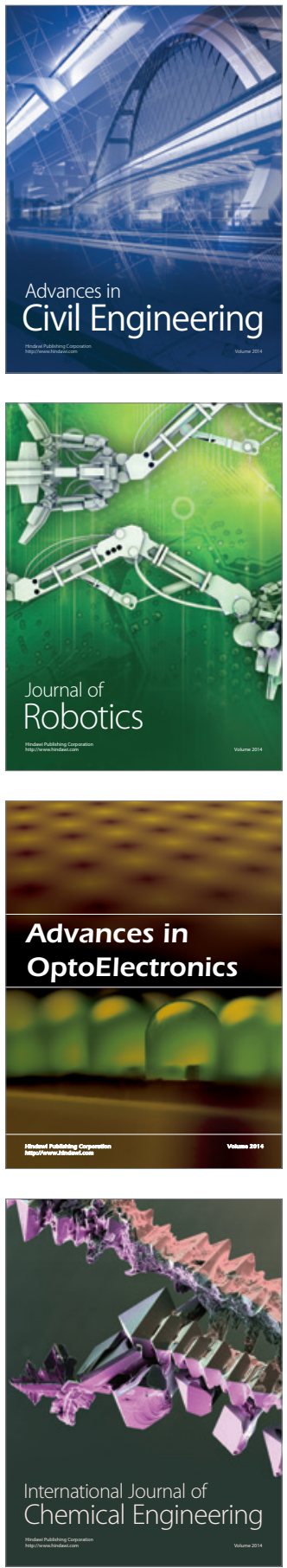

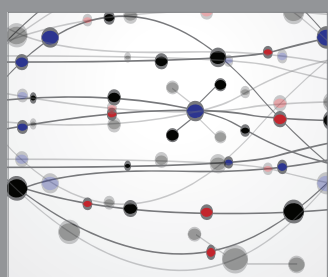

The Scientific World Journal

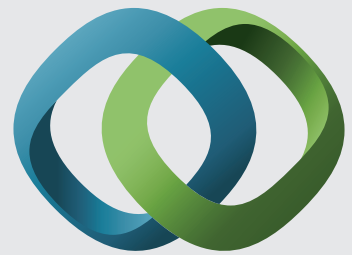

\section{Hindawi}

Submit your manuscripts at

http://www.hindawi.com
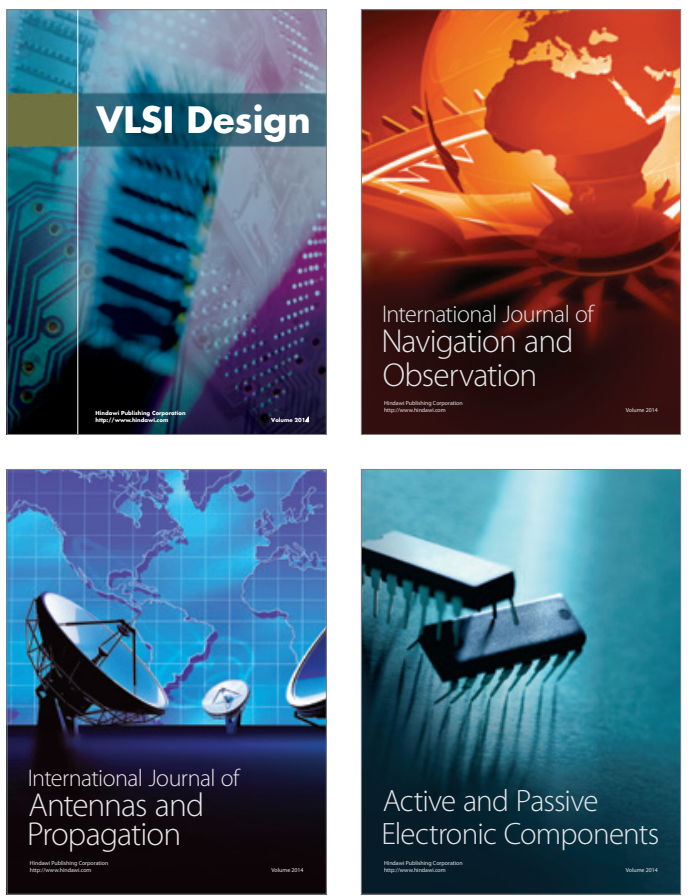
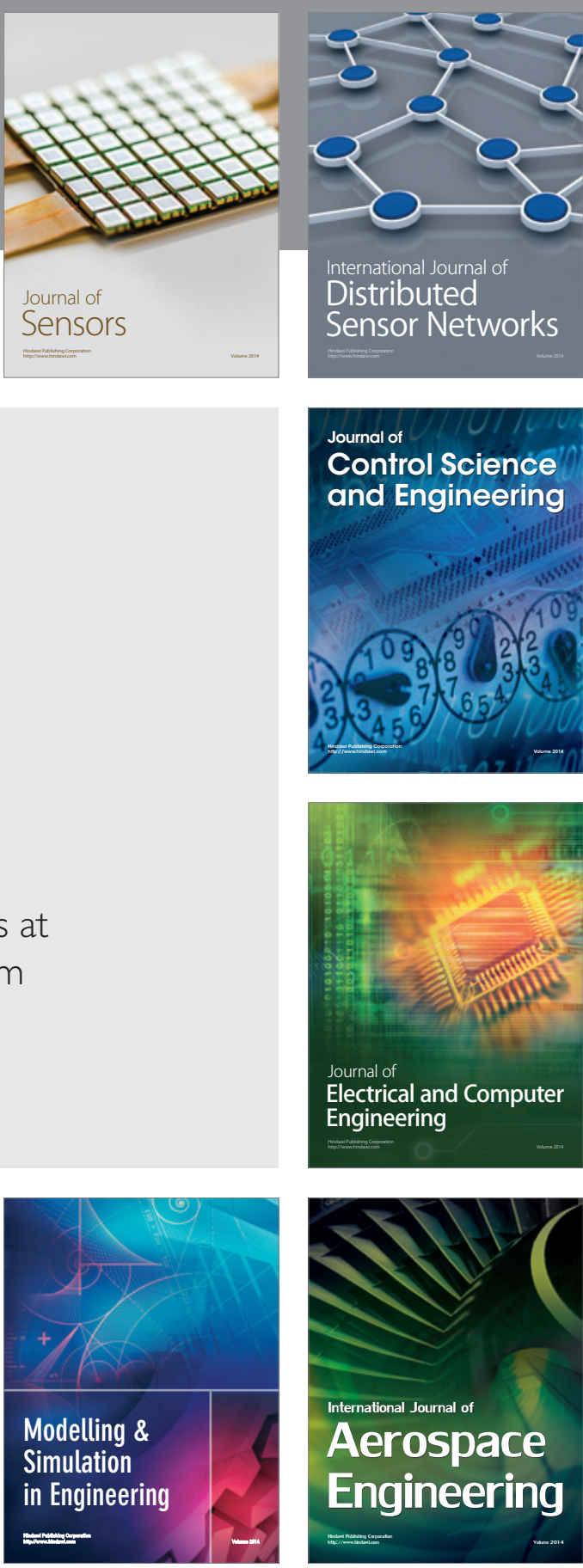

International Journal of

Distributed

Sensor Networks

Journal of

Control Science

and Engineering
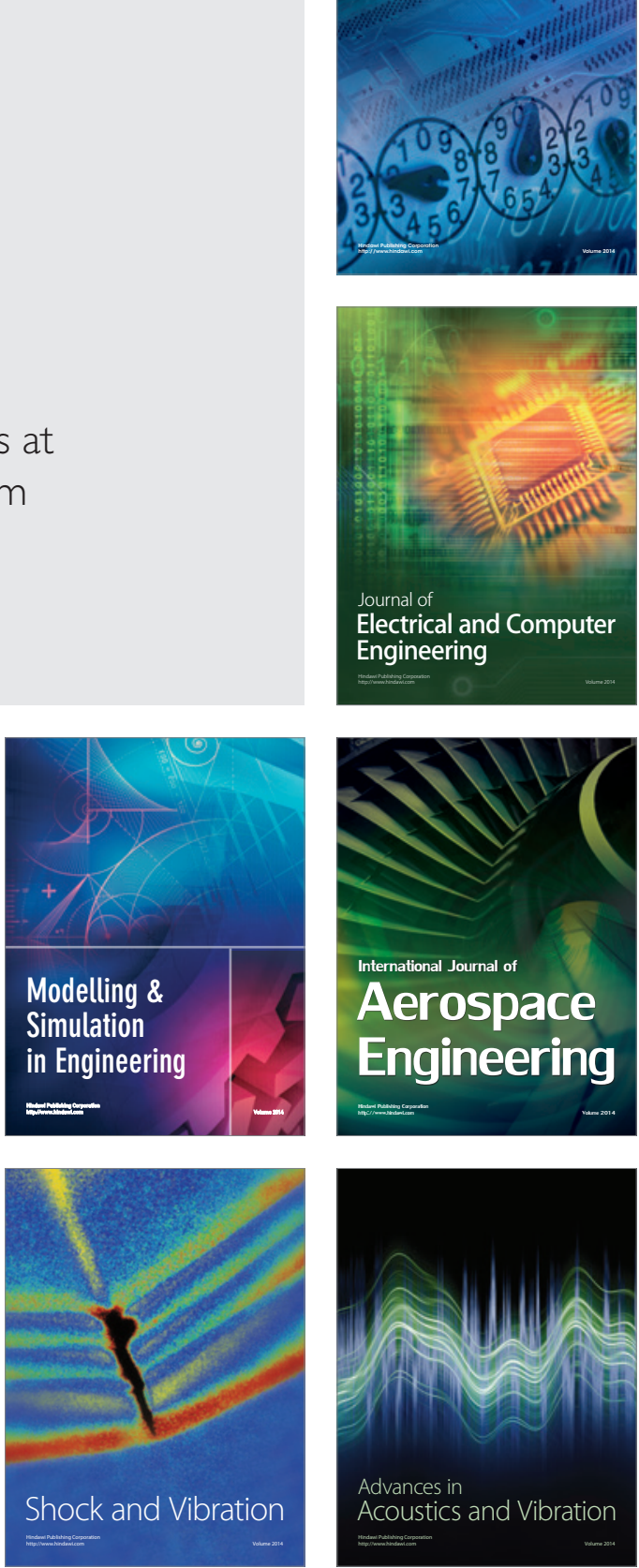